\title{
Sugars within a Hydrophobic Scaffold: Glycodendrimers from Polyphenylenes
}

Junji Sakamoto and Klaus Müllen*

Max-Planck-Institute for Polymer Research, Ackermannweg 10, 55128 Mainz, Germany 


\section{Supporting Information}

Synthetic procedure to 4 and $\mathbf{1 0}$

\section{Scheme S1}
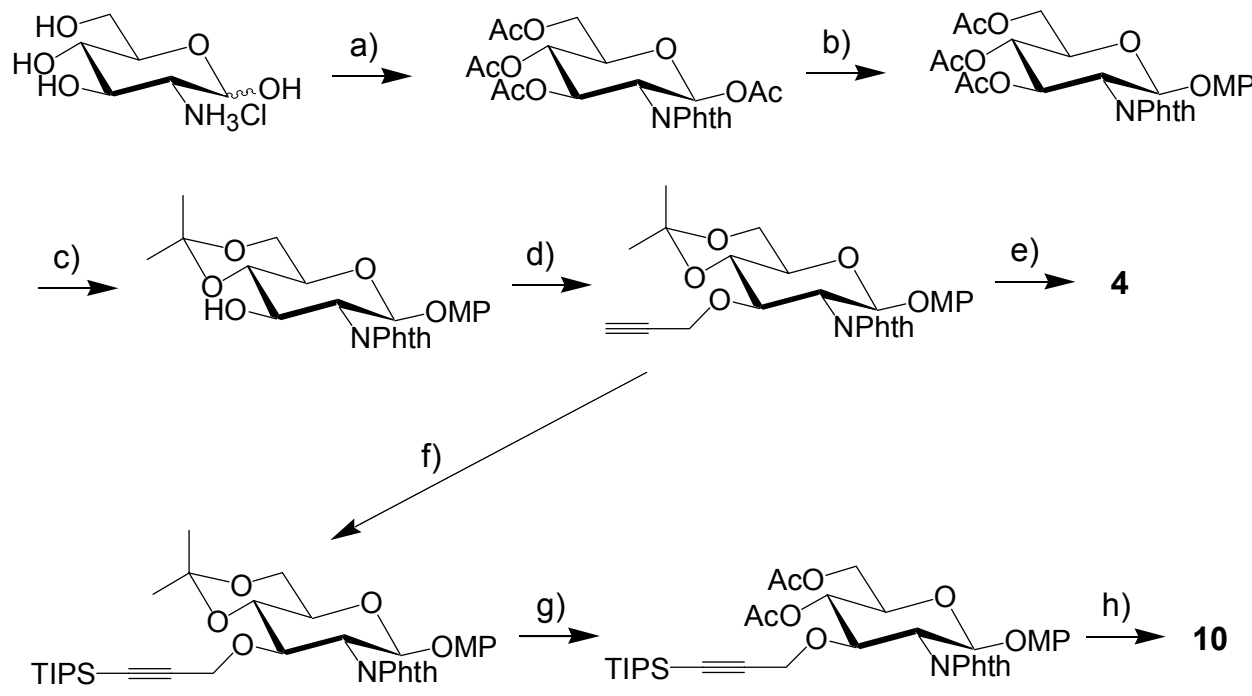

a) (i) $\mathrm{NaOMe} / \mathrm{MeOH}$ (ii) Phthalic anhydride, $\mathrm{NEt} / \mathrm{MeOH}$ (iii) $\mathrm{Ac}_{2} \mathrm{O}$, DMAP/Py, 59\% (3 steps). b) p-Methoxyphenol, TMSOTf/ $\mathrm{CH}_{2} \mathrm{ClCH}_{2} \mathrm{Cl}, 73 \%$. c) (i) $\mathrm{NaOMe} / \mathrm{MeOH}$ (ii) $\mathrm{Me}{ }_{2} \mathrm{C}(\mathrm{OMe})_{2}$, TsOH/DMF, 84\% (2 steps). d) Propargyl bromide, NaH/DMF, 60\%. e) (i) $\mathrm{TsOH} / \mathrm{MeOH}$ (ii) $\mathrm{Ac}_{2} \mathrm{O}, \mathrm{DMAP} / \mathrm{Py}, 93 \%$ (2 steps). f) TIPSCl, $n$-BuLi $/ \mathrm{THF}, 22 \%$. g) (i) $\mathrm{TsOH} / \mathrm{MeOH}$ (ii) $\mathrm{Ac}_{2} \mathrm{O}$, DMAP/Py, $97 \%$ (2 steps). h) (i) CAN/toluene-MeCN-water 4:3:3 (ii) $\mathrm{CCl}_{3} \mathrm{CN}, \mathrm{DBU} / \mathrm{CH}_{2} \mathrm{Cl}_{2}$ at $0{ }^{\circ} \mathrm{C}, 54 \%$ (2 steps).

\section{Spectroscopic data of key compounds $(3,5,7,9,11,13,14,17,18,19,21,22)$}

3: ${ }^{1} \mathrm{H}$ NMR (250 MHz, $\mathrm{CDCl}_{3}$ ): $\delta$ 7.80-7.61 (br, 8H, 2 Phth), 7.27-7.11 (m, 10H, $\left.2 \mathrm{C}_{6} \mathrm{H}_{5}\right), 6.85$ and $6.61\left(2 \mathrm{~d}\right.$, each $\left.4 \mathrm{H}, 2 \mathrm{C}_{6} \mathrm{H}_{4}\right), 5.78(\mathrm{dd}, 2 \mathrm{H}, 2 \mathrm{H} 3), 5.33(\mathrm{~d}, 2 \mathrm{H}, 2 \mathrm{H} 1), 5.17(\mathrm{dd}, 2 \mathrm{H}, 2 \mathrm{H} 4)$, 4.78 and $4.50\left(2 \mathrm{~d}\right.$, each $\left.2 \mathrm{H}, 2 \mathrm{C} 1-\mathrm{OCH}_{2}\right), 4.41-4.26(\mathrm{~m}, 4 \mathrm{H}, 2 \mathrm{H} 2$ and $2 \mathrm{H} 6 \mathrm{a}), 4.16(\mathrm{dd}, 2 \mathrm{H}, 2$ H6b), 3.82 (ddd, 2H, $2 \mathrm{H} 5$ ), 2.08, 2.02, and 1.85 (3 s, each $\left.6 \mathrm{H}, 3 \mathrm{OC}(=\mathrm{O}) \mathrm{CH}_{3}\right) ; \mathrm{J}_{1,2}=8.5 \mathrm{~Hz}, \mathrm{~J}_{2,3}$ $=10.1 \mathrm{~Hz}, \mathrm{~J}_{3,4}=9.2 \mathrm{~Hz}, \mathrm{~J}_{4,5}=10.4 \mathrm{~Hz}, \mathrm{~J}_{5,6 \mathrm{a}}=4.1 \mathrm{~Hz}, \mathrm{~J}_{5,6 \mathrm{~b}}=2.5 \mathrm{~Hz}, \mathrm{~J}_{6 \mathrm{a}, 6 \mathrm{~b}}=12.3 \mathrm{~Hz} ;{ }^{13} \mathrm{C} \mathrm{NMR}$ $\left(63 \mathrm{MHz}, \mathrm{CDCl}_{3}\right): \delta 200.1(\mathrm{C}=\mathrm{O}$ of cyclopentadieneone), 170.6, 170.1 and 169.5 $\left(\mathrm{OC}(=\mathrm{O}) \mathrm{CH}_{3}\right), 167.5,134.3,131.3$ and 123.6 (Phth), 153.7, 137.1, 132.4, 130.5, 130.1, 129.2, 128.1, 127.5, 127.0 and 125.3 (the other aromatic carbons), 97.0 (C1), 71.9 (C5), 70.6 (C3), $70.3\left(\mathrm{C} 1-\mathrm{OCH}_{2}\right), 68.9(\mathrm{C} 4), 61.9(\mathrm{C} 6), 54.5(\mathrm{C} 2), 20.7,20.6$, and $20.4\left(\mathrm{OC}(=\mathrm{O}) \underline{\mathrm{CH}}_{3}\right)$; 
MALDI-TOF-MS (2,5-dihyroxybenzoic acid) calcd for $\mathrm{C}_{71} \mathrm{H}_{62} \mathrm{~N}_{2} \mathrm{O}_{21} ; 1278.4,1279.4,1280.4$, 1281.4, 1282.4; found: $\mathrm{m} / \mathrm{z} 1279,1280,1281,1282,1283\left(\sim[\mathrm{M}+\mathrm{H}]^{+}\right), 1302,1303,1304$, $1305,1306\left(\sim[\mathrm{M}+\mathrm{Na}]^{+}\right), 1318,1319,1320,1321,1322\left(\sim[\mathrm{M}+\mathrm{K}]^{+}\right)$.

5: ${ }^{1} \mathrm{H}$ NMR $\left(250 \mathrm{MHz}, \mathrm{CDCl}_{3}\right): \delta$ 7.82-7.47 (m, 13H, 3 Phth and $\left.\mathrm{C}_{6} \mathrm{H}\right), 6.76$ and $6.65(2 \mathrm{~d}$, each $\left.4 \mathrm{H}, \mathrm{C} 1-\mathrm{OC}_{6} \underline{\mathrm{H}}_{4} \mathrm{OCH}_{3}\right), 7.18-6.25(\mathrm{~m}, 18 \mathrm{H}$, the other aromatic protons), 5.71 and $5.68(2 \mathrm{dd}, 2 \mathrm{H}$, H3' and $\left.\mathrm{H}^{\prime}{ }^{*}\right), 5.55(\mathrm{~d}, 1 \mathrm{H}, \mathrm{H} 1), 5.20-5.03\left(\mathrm{~m}, 3 \mathrm{H}, \mathrm{H} 4, \mathrm{H} 4{ }^{\prime}\right.$ and $\left.\mathrm{H} 4{ }^{\prime}{ }^{*}\right), 5.02$ and $4.91(2 \mathrm{~d}, 2 \mathrm{H}$, $\mathrm{H} 1$ ' and $\left.\mathrm{H} 1{ }^{\prime}{ }^{*}\right), 4.59-4.01\left(\mathrm{~m}, 16 \mathrm{H}, \mathrm{H} 2, \mathrm{H} 3, \mathrm{C} 3-\mathrm{OCH}_{2}, \mathrm{C}^{\prime}{ }^{\prime}-\mathrm{OCH}_{2}, \mathrm{C} 1{ }^{\prime} *-\mathrm{OCH}_{2}, \mathrm{H} 2\right.$ ', $\mathrm{H} 2{ }^{\prime} *$, H6a, H6b, H6a', H6b', H6a'* and H6b'*), 3.76 (ddd, 1H, H5), 3.64 (s, 3H, C1-OC ${ }_{6} \mathrm{H}_{4} \mathrm{OC}{ }_{3}$ ), 3.61-3.45 (m, 2H, H5' and $\left.\mathrm{H} 5^{\prime}{ }^{*}\right), 2.034,2.028,2.016,2.009,1.99,1.81$ and $1.65(7 \mathrm{~s}, 24 \mathrm{H}$, $\left.\mathrm{OC}(=\mathrm{O}) \mathrm{CH}_{3}\right) ; \mathrm{J}_{1,2}=8.2 \mathrm{~Hz}, \mathrm{~J}_{1^{\prime}, 2},=\mathrm{J}_{1^{\prime}, 2^{2} *}=8.5 \mathrm{~Hz} ;{ }^{13} \mathrm{C} \mathrm{NMR}\left(63 \mathrm{MHz}, \mathrm{CDCl}_{3}\right): 170.53,170.46$, 170.0, 169.4 and $169.2\left(\mathrm{O} \underline{\mathrm{C}}(=\mathrm{O}) \mathrm{CH}_{3}\right), 167.2,134.1,131.2$ and 123.3 (Phth), 155.4, 150.5, $141.0,140.7,140.5,139.3,139.2,138.4,134.9,133.1,132.7,131.04,130.95,130.7,129.9$, $129.8,129.7,128.8,127.5,127.1,126.4,126.2,126.1,126.0,118.5$ and 114.2 (the other aromatic carbons), $97.6(\mathrm{C} 1), 95.9$ and $95.7\left(\mathrm{C} 1^{\prime}\right.$ and $\left.\mathrm{C} 1^{\prime *}\right), 77.3(\mathrm{C} 3), 72.5\left(\mathrm{C} 3-\mathrm{OCH}_{2}\right), 72.2$ (C5), 71.6 (C5' and $\left.\mathrm{C}^{\prime} *\right), 71.3(\mathrm{C} 4), 70.54$ and 70.51 (C3' and $\left.\mathrm{C} 3^{\prime}{ }^{*}\right), 70.0$ and 69.9

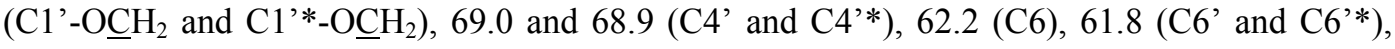
$55.4\left(\mathrm{C} 1-\mathrm{OC}_{6} \mathrm{H}_{4} \mathrm{OCH}_{3}\right), 55.3(\mathrm{C} 2), 54.31$ and 54.27 (C2' and $\left.\mathrm{C} 2{ }^{\prime} *\right), 20.63,20.60,20.53,20.49$ and $20.3\left(\mathrm{OC}(=\mathrm{O}) \underline{\mathrm{CH}}_{3}\right)$; MALDI-TOF-MS (2,5-dihyroxybenzoic acid) calcd for $\mathrm{C}_{98} \mathrm{H}_{89} \mathrm{~N}_{3} \mathrm{O}_{30}$; 1787.6, 1788.6, 1789.6, 1790.6, 1791.6, 1792.6; found: $\mathrm{m} / \mathrm{z} 1811,1812,1813,1814,1815$ ( $\left.[\mathrm{M}+\mathrm{Na}]^{+}\right), 1827,1828,1829,1830,1831\left(\sim[\mathrm{M}+\mathrm{K}]^{+}\right)$.

7: ${ }^{1} \mathrm{H}$ NMR (250 MHz, $\left.\mathrm{CDCl}_{3}\right): \delta 7.82-7.42\left(\mathrm{~m}, 26 \mathrm{H}, 6\right.$ Phth and $\left.2 \mathrm{C}_{6} \mathrm{H}\right), 7.26-6.26(\mathrm{~m}, 54 \mathrm{H}$, the other aromatic protons), 5.73 and 5.69 (2 dd, 4H, 2 H3' and 2 H3'*), 5.18-4.89 (m, 12H, 2 H4', $2 \mathrm{H} 4^{\prime} *, 2 \mathrm{H} 4,2 \mathrm{H} 1,2 \mathrm{H}{ }^{\prime}$ and $\left.2 \mathrm{H} 1^{\prime} *\right), 4.78-4.01$ (m, 36H, $2 \mathrm{C} 1-\mathrm{OCH}_{2}, 2 \mathrm{C} 3-\mathrm{OCH}_{2}, 2$ C1'- $\mathrm{OCH}_{2}, 2$ C1'*-OCH 2,2 H3, 2 H2, 2 H2', 2 H2'*, 2 H6a, 2 H6a', 2 H6a'*, 2 H6b, 2 H6b' \left. and $2{\mathrm{H} 6 \mathrm{~b}^{\prime}}^{*}\right), 3.70-3.47\left(\mathrm{~m}, 6 \mathrm{H}, 2 \mathrm{H} 5,2 \mathrm{H} 5^{\prime}\right.$ and $\left.2 \mathrm{H} 5^{\prime} *\right), 2.04,2.03,1.83$, and $1.66(4 \mathrm{~s}, 48 \mathrm{H}$, $\left.\mathrm{OC}(=\mathrm{O}) \mathrm{CH}_{3}\right) ; \mathrm{J}_{1,2}=\mathrm{J}_{1^{\prime}, 2},=\mathrm{J}_{1^{*}, 2^{* *}}=8.5 \mathrm{~Hz} ;{ }^{13} \mathrm{C} \mathrm{NMR}\left(63 \mathrm{MHz}, \mathrm{CDCl}_{3}\right): \delta 200.1(\underline{\mathrm{C}}=\mathrm{O}$ of cyclopentadieneone), 170.6, 170.5, 170.0, 169.4 and $169.3\left(\mathrm{OC}(=\mathrm{O}) \mathrm{CH}_{3}\right), 167.3,134.2,134.0$, 131.3 and 123.4 (Phth), 153.7, 141.1, 140.7, 140.5, 139.4, 139.3, 139.2, 138.5, 137.3, 135.0, $133.2,132.8,132.2,131.1,131.0,130.8,130.5,130.0,129.83,129.75,129.0,128.8,128.1$, $127.6,127.4,127.2,126.7,126.4,126.2,126.0$ and 125.2 (the other aromatic carbons), 97.2 (C1), 96.1 and $95.9\left(\mathrm{Cl}^{\prime}\right.$ and $\left.\mathrm{C} 1^{\prime *}\right), 77.4(\mathrm{C} 3), 72.4\left(\mathrm{C} 3-\mathrm{OCH}_{2}\right), 72.2(\mathrm{C} 5), 71.6\left(\mathrm{C} 5{ }^{\prime}\right.$ and $\left.\mathrm{C}^{\prime}{ }^{*}\right), 71.3(\mathrm{C} 4), 70.63$ and $70.59\left(\mathrm{C} 3^{\prime}\right.$ and $\left.\mathrm{C} 3^{*}{ }^{*}\right), 70.12$ and $70.08\left(\mathrm{C}^{\prime}{ }^{\prime}-\mathrm{OCH}_{2}\right.$ and $\left.\mathrm{C}{ }^{*}{ }^{*}-\mathrm{OCH}_{2}\right), 69.9\left(\mathrm{C} 1-\mathrm{OCH}_{2}\right), 69.92$ and $68.99\left(\mathrm{C} 4{ }^{\prime}\right.$ and $\left.\mathrm{C} 4{ }^{*}\right), 62.3(\mathrm{C} 6), 61.8\left(\mathrm{C} 6{ }^{\prime}\right.$ and $\left.\mathrm{C}^{\prime *}\right), 55.4$ (C2), 54.40 and 54.36 (C2' and $\left.\mathrm{C}^{\prime}{ }^{*}\right), 20.73,20.68,20.59,20.56$ and 20.4 
$\left(\mathrm{OC}(=\mathrm{O}) \underline{\mathrm{CH}}_{3}\right)$; MALDI-TOF-MS (2,5-dihyroxybenzoic acid) calcd for $\mathrm{C}_{213} \mathrm{H}_{186} \mathrm{~N}_{6} \mathrm{O}_{59} ; 3771.2$, 3772.2, 3773.2, 3774.2, 3775.2, 3776.2, 3777.2, 3778.2, 3779.2; found: $\mathrm{m} / \mathrm{z} 3796,3797,3798$, 3799, 3800, 3801, $3802\left(\sim[\mathrm{M}+\mathrm{Na}]^{+}\right), 3812,3813,3814,3815,3816,3817,3818,3819,3820$, $3821,3822\left(\sim[\mathrm{M}+\mathrm{K}]^{+}\right)$.

9: ${ }^{1} \mathrm{H}$ NMR (500 MHz, $\mathrm{CDCl}_{3}$ ): $\delta$ 7.84-7.64 (br, 96H, 24 Phth) 7.64-7.40 (br, $12 \mathrm{H}, 12 \mathrm{C}_{6} \mathrm{H}$ ), 7.21-6.27 (m, 232 $\mathrm{H}$, the other aromatic protons), 5.77-5.66 (m, 16H, $4 \mathrm{H3}$ ', $4 \mathrm{H3}{ }^{\prime} *, 4 \mathrm{H} 3^{\text {, * }}$ and

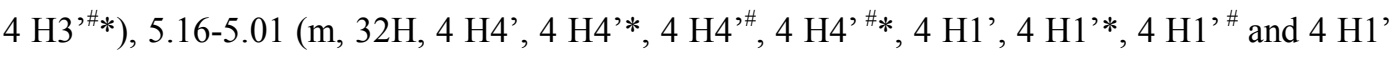
\#*), 4.99-4.83 (m, 16H, $4 \mathrm{H} 1,4 \mathrm{H1}^{\#}, 4 \mathrm{H} 4$ and $\left.4 \mathrm{H} 4^{\#}\right), 4.63-3.99\left(\mathrm{~m}, 144 \mathrm{H}, 4 \mathrm{C} 1-\mathrm{OCH}_{2}, 4\right.$ $\mathrm{Cl}^{\#}-\mathrm{OCH}_{2}, 4 \mathrm{Cl}{ }^{\prime}-\mathrm{OCH}_{2}, 4 \mathrm{Cl}{ }^{\prime}{ }_{-}-\mathrm{OCH}_{2}, 4 \mathrm{Cl}{ }^{\text {, }}-\mathrm{OCH}_{2}, 4 \mathrm{Cl}{ }^{\text {, }}{ }^{*}-\mathrm{OCH}_{2}, 4 \mathrm{C} 3-\mathrm{OCH}_{2}, 4$

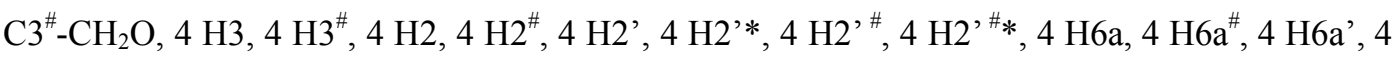
H6a'*, 4 H6a " , 4 H6a "\#*, 4 H6b, 4 H6b , 4 H6b', 4 H6b'*, 4 H6b' ${ }^{\text {* }}$ and 4 H6b' **), 3.65-3.41

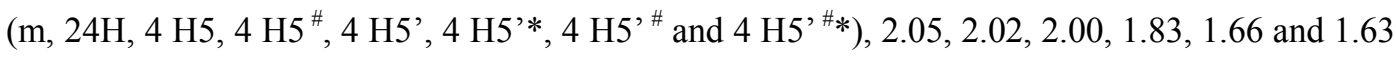
$\left(6 \mathrm{~s}, 192 \mathrm{H}, \mathrm{OC}(=\mathrm{O}) \underline{\mathrm{H}}_{3}\right) ;{ }^{13} \mathrm{C} \mathrm{NMR}\left(125 \mathrm{MHz}, \mathrm{CDCl}_{3}\right): \delta 170.5,170.0,169.4$ and 169.3 $\left(\mathrm{OC}(=\mathrm{O}) \mathrm{CH}_{3}\right), 167.3,134.2,131.4$ and 123.4 (Phth), 144.2, 141.5, 141.2, 140.9, 140.7, 140.6, $139.7,139.4,139.3,139.2,138.7,138.5,135.0,134.0,133.3,132.9,131.5,131.1,130.8,130.3$, $130.0,129.83,129.77,128.8,128.5,127.6,127.2,126.7,126.4,126.2$ and 126.0 (the other aromatic carbons), 96.9 and $96.7\left(\mathrm{C} 1\right.$ and $\left.\mathrm{C} 1^{\#}\right), 96.21,96.15,96.10$ and 96.03 (C1', $\mathrm{C}{ }^{\prime}{ }^{*}, \mathrm{C} 1$ ' \# and $\left.\mathrm{C} 1^{* *}\right), 77.47$ and $77.40\left(\mathrm{C} 3\right.$ and $\left.\mathrm{C}^{*}\right), 72.3\left(\mathrm{C} 3-\mathrm{OCH}_{2}\right.$ and $\left.\mathrm{C}^{\#}-\mathrm{CH}_{2}\right), 72.1\left(\mathrm{C} 5\right.$ and $\left.\mathrm{C}^{*}\right)$,

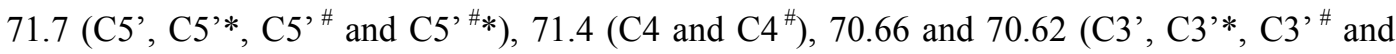

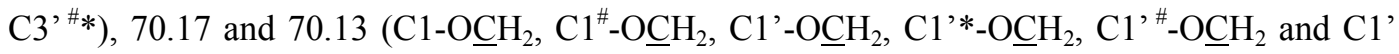

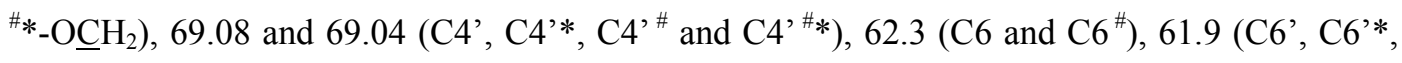

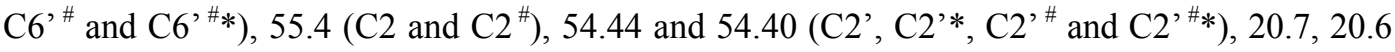
and $20.4\left(\mathrm{OC}(=\mathrm{O}) \underline{\mathrm{CH}}_{3}\right) ;$ MALDI-TOF-MS (dithranol) calcd for $\mathrm{C}_{881} \mathrm{H}_{764} \mathrm{~N}_{24} \mathrm{O}_{232} ; 15400$ (averaged); found: m/z 15406 .

11: ${ }^{1} \mathrm{H}$ NMR (250 MHz, $\mathrm{CDCl}_{3}$ ): $\delta$ 7.80-7.55 (br, 8H, 2 Phth), 7.27-7.09 (m, 10H, $2 \mathrm{C}_{6} \mathrm{H}_{5}$ ), 6.81 and $6.56\left(2 \mathrm{~d}\right.$, each $\left.4 \mathrm{H}, 2 \mathrm{C}_{6} \mathrm{H}_{4}\right), 5.15(\mathrm{~d}, 2 \mathrm{H}, 2 \mathrm{H1}), 5.12(\mathrm{dd}, 2 \mathrm{H}, 2 \mathrm{H} 4), 4.75$ and $4.46(2 \mathrm{~d}$, each $\left.2 \mathrm{H}, 2 \mathrm{C} 1-\mathrm{OCH}_{2}\right), 4.62(\mathrm{dd}, 2 \mathrm{H}, 2 \mathrm{H} 3), 4.36-4.21$ (m, 4H, $2 \mathrm{H} 2$ and $\left.2 \mathrm{H6a}\right), 4.16$ and 4.15 (d, 4H, C3- $\mathrm{OCH}_{2}$ ), 4.10 (dd, 2H, $2 \mathrm{H6b}$ ), 3.69 (ddd, 2H, $2 \mathrm{H} 5$ ), 2.09, and 2.06 (2 s, each 6H, $\mathrm{OC}(=\mathrm{O}) \mathrm{CH}_{3}$ ), 0.97-0.87 (m, 42H, 2 TIPS); $\mathrm{J}_{1,2}=8.5 \mathrm{~Hz}, \mathrm{~J}_{2,3}=10.4 \mathrm{~Hz}, \mathrm{~J}_{3,4}=8.8 \mathrm{~Hz}, \mathrm{~J}_{4,5}=10.4$ $\mathrm{Hz}, \mathrm{J}_{5,6 \mathrm{a}}=4.4 \mathrm{~Hz}, \mathrm{~J}_{5,6 \mathrm{~b}}=2.2 \mathrm{~Hz} ;{ }^{13} \mathrm{C} \mathrm{NMR}\left(63 \mathrm{MHz}, \mathrm{CDCl}_{3}\right): \delta 200.1(\mathrm{C}=\mathrm{O}$ of cyclopentadieneone), 170.7 and $\left.169.4(\mathrm{O} \underline{(}(=)) \mathrm{CH}_{3}\right), 167.7,134.1,131.5$ and 123.5 (Phth), $153.8,137.3,132.2,130.5,130.1,129.1,128.1,127.4,126.8$ and 125.2 (the other aromatic carbons), 101.9 (C3-OCH 2 C $\equiv \mathrm{C}-\mathrm{TIPS}), 97.3$ (C1), 88.4 (C3-OCH $\left.{ }_{2} \mathrm{C} \equiv \mathrm{C}-\mathrm{TIPS}\right), 75.5$ (C3), 72.1 (C5), $70.7(\mathrm{C} 4), 70.1\left(\mathrm{C} 1-\mathrm{OCH}_{2}\right), 62.2(\mathrm{C} 6), 59.4\left(\mathrm{C} 3-\mathrm{OCH}_{2}\right), 55.3(\mathrm{C} 2), 20.9$ and 20.7 
$\left(\mathrm{OC}(=\mathrm{O}) \underline{\mathrm{CH}}_{3}\right), 18.4\left(\mathrm{CH}\left(\underline{\mathrm{CH}}_{3}\right)_{2}\right), 11.0\left(\underline{\mathrm{CH}}\left(\mathrm{CH}_{3}\right)_{2}\right)$; MALDI-TOF-MS (2,5-dihyroxybenzoic acid) calcd for $\mathrm{C}_{91} \mathrm{H}_{102} \mathrm{~N}_{2} \mathrm{O}_{19} \mathrm{Si}_{2} ; 1582.7,1583.7,1584.7,1585.7,1586.7,1587.7$; found: $\mathrm{m} / \mathrm{z}$ $1583,1584,1585,1586,1587,1588\left(\sim[\mathrm{M}+\mathrm{H}]^{+}\right) ; 1606,1607,1608,1609,1610,1611(\sim[\mathrm{M}+$ $\left.\mathrm{Na}]^{+}\right) ; 1622,1623,1624,1625,1626,1627\left(\sim[\mathrm{M}+\mathrm{K}]^{+}\right)$.

13: ${ }^{1} \mathrm{H}$ NMR (250 MHz, $\mathrm{CDCl}_{3}$ ): $\delta$ 7.92-7.56 (br, 4H, 2 DCPhth), 7.29-7.10 (m, 10H, $2 \mathrm{C}_{6} \mathrm{H}_{5}$ ), 6.88 and $6.66\left(2 \mathrm{~d}\right.$, each $\left.4 \mathrm{H}, 2 \mathrm{C}_{6} \mathrm{H}_{4}\right), 5.70(\mathrm{dd}, 2 \mathrm{H}, 2 \mathrm{H} 3), 5.28(\mathrm{~d}, 2 \mathrm{H}, 2 \mathrm{H} 1), 5.17(\mathrm{dd}, 2 \mathrm{H}, 2$ $\mathrm{H} 4), 4.79$ and 4.51 ( $2 \mathrm{~d}$, each $\left.2 \mathrm{H}, 2 \mathrm{C} 1-\mathrm{OCH}_{2}\right), 4.37-4.23$ (m, 4H, $2 \mathrm{H} 2$ and $\left.2 \mathrm{H} 6 \mathrm{a}\right), 4.16$ (dd, $2 \mathrm{H}, 2 \mathrm{H6b}$ ), 3.80 (ddd, 2H, $2 \mathrm{H} 5), 2.07,2.02$ and 1.85 (3 s, each $\left.6 \mathrm{H}, \mathrm{OC}(=\mathrm{O}) \mathrm{CH}_{3}\right) ; \mathrm{J}_{1,2}=8.5$ $\mathrm{Hz}, \mathrm{J}_{2,3}=10.5 \mathrm{~Hz}, \mathrm{~J}_{3,4}=9.2 \mathrm{~Hz}, \mathrm{~J}_{4,5}=10.1 \mathrm{~Hz}, \mathrm{~J}_{5,6 \mathrm{a}}=4.4 \mathrm{~Hz}, \mathrm{~J}_{5,6 \mathrm{~b}}=2.5 \mathrm{~Hz}, \mathrm{~J}_{6 \mathrm{a}, 6 \mathrm{~b}}=12.3 \mathrm{~Hz} ;{ }^{13} \mathrm{C}$ NMR (63 MHz, $\left.\mathrm{CDCl}_{3}\right): \delta 199.9(\mathrm{C}=\mathrm{O}$ of cyclopentadieneone), 170.5, 170.2 and 169.3 $\left(\mathrm{O} \underline{\mathrm{C}}(=\mathrm{O}) \mathrm{CH}_{3}\right), 165.4,139.3,130.5$ and 125.6 (DCPhth), 153.4, 136.9, 132.6, 130.4, 130.1, 129.3, 128.1, 127.5 and 127.1 (the other aromatic carbons), 96.6 (C1), 71.9 (C5), 70.6 (C3), $70.2\left(\mathrm{C} 1-\mathrm{OCH}_{2}\right), 68.7(\mathrm{C} 4), 61.9(\mathrm{C} 6), 54.9(\mathrm{C} 2), 20.7,20.6$ and $20.4\left(\mathrm{OC}(=\mathrm{O}) \underline{\mathrm{CH}}_{3}\right)$; MALDI-TOF-MS (2,5-dihyroxybenzoic acid) calcd for $\mathrm{C}_{71} \mathrm{H}_{58} \mathrm{Cl}_{4} \mathrm{~N}_{2} \mathrm{O}_{21} ; 1414.2,1415.2,1416.2$, 1417.2, 1418.2, 1419.2, 1420.2, 1421.2, 1422.2, 1423.2; found: $\mathrm{m} / \mathrm{z}$ 1415, 1416, 1417, 1418, $1419,1420,1421,1422,1423,1424\left(\sim[\mathrm{M}+\mathrm{H}]^{+}\right) ; 1438,1439,1440,1441,1442,1443,1444$, $1445,1446,1447\left(\sim[\mathrm{M}+\mathrm{Na}]^{+}\right) ; 1453,1454,1455,1456,1457,1458,1459,1460,1461,1462$ $\left(\sim[\mathrm{M}+\mathrm{K}]^{+}\right)$.

14: ${ }^{1} \mathrm{H}$ NMR (250 MHz, $\mathrm{CDCl}_{3}$ ): $\delta$ 7.88-7.60 (m, 32H, 8 Phth), 7.54 (s, 4H, $4 \mathrm{C}_{6} \mathrm{H}$ ), 7.22-6.41 (m, $88 \mathrm{H}$, the other aromatic protons), 5.11 and $5.06(2 \mathrm{dd}, 8 \mathrm{H}, 4 \mathrm{H} 4$ and $4 \mathrm{H} 4 *), 4.97$ and 4.93 $\left(2 \mathrm{~d}, 8 \mathrm{H}, 4 \mathrm{H} 1\right.$ and $\left.4 \mathrm{H} 1^{*}\right), 4.66-4.01\left(\mathrm{~m}, 64 \mathrm{H}, 4 \mathrm{H} 3,4 \mathrm{H} 3 *\right.$, $4 \mathrm{C} 1-\mathrm{OCH}_{2}, 4 \mathrm{C} 1-\mathrm{OCH}_{2}{ }^{*}, 4 \mathrm{H} 2,4$ H2*, 4 H6a, 4 H6a*, 4 C3- $-\mathrm{OCH}_{2}, 4$ C3*-OCH, 4 H6b and 4 H6b*), 3.59-3.45 (m, 8H, 4 H5 and $\left.4 \mathrm{H}^{*}\right), 2.11,2.07,2.06$ and $2.04\left(4 \mathrm{~s}\right.$, each $\left.12 \mathrm{H}, \mathrm{OC}(=\mathrm{O}) \mathrm{CH}_{3}\right), 0.99-0.82(\mathrm{~m}, 168 \mathrm{H}$, $\mathrm{CH}\left(\mathrm{C}_{3}\right)_{2}, \mathrm{CH}\left(\underline{\mathrm{C}}_{3}\right)_{2}{ }^{*}, \mathrm{C} \underline{\mathrm{H}}\left(\mathrm{CH}_{3}\right)_{2}$ and $\left.\mathrm{C} \underline{\mathrm{H}}\left(\mathrm{CH}_{3}\right)_{2}{ }^{*}\right) ; \mathrm{J}_{1,2}=\mathrm{J}_{1^{*} 2^{*}}=8.2 \mathrm{~Hz} ;{ }^{13} \mathrm{C} \mathrm{NMR}(63 \mathrm{MHz}$, $\left.\mathrm{CDCl}_{3}\right): \delta 170.6$ and $169.3\left(\mathrm{OC}(=\mathrm{O}) \mathrm{CH}_{3}\right), 167.6,134.0,131.5$ and 123.4 (Phth and Phth*), 144.3, 141.4, 140.9, 140.7, 140.6, 139.7, 139.5, 139.4, 139.2, 138.6, 133.6 133.4, 131.2, 130.3, $129.9,128.5,127.7,126.9,126.7,126.2,125.9$ and 125.4 (the other aromatic carbons), 101.9 $\left(\mathrm{C} 3-\mathrm{OCH}_{2} \underline{\mathrm{C}} \equiv \mathrm{CTIPS}\right.$ and $\left.\mathrm{C}^{*}-\mathrm{OCH}_{2} \underline{\mathrm{C}} \equiv \mathrm{CTIPS}\right), 96.7$ and $96.5(\mathrm{C} 1$ and $\mathrm{C} 1 *), 88.2$ $\left(\mathrm{C} 3-\mathrm{OCH}_{2} \mathrm{C} \equiv \underline{\mathrm{C}}\right.$-TIPS and $\left.\mathrm{C} 3 *-\mathrm{OCH}_{2} \mathrm{C} \equiv \underline{\mathrm{C}}-\mathrm{TIPS}\right), 75.7$ and 75.6 (C3 and $\left.\mathrm{C} 3 *\right), 72.0$ (C5 and $\left.\mathrm{C}^{*}\right), 70.7$ ( $\mathrm{C} 4$ and $\left.\mathrm{C} 4 *\right), 70.2$ and $70.0\left(\mathrm{C} 1-\mathrm{OCH}_{2}\right), 62.1$ (C6 and $\left.\mathrm{C} 6 *\right), 59.31$ and 59.26 $\left(\mathrm{C} 3-\mathrm{OCH}_{2}\right.$ and $\left.\mathrm{C} 3 *-\underline{\mathrm{CH}}_{2}\right), 55.2$ and $55.1(\mathrm{C} 2$ and $\mathrm{C} 2 *), 20.9$ and $20.7\left(\mathrm{OC}(=\mathrm{O}) \underline{\mathrm{CH}}_{3}\right), 18.4$ $\left(-\mathrm{CH}\left(\underline{\mathrm{CH}}_{3}\right)_{2}\right), 11.0\left(-\underline{\mathrm{C}} \mathrm{H}\left(\mathrm{CH}_{3}\right)_{2}\right)$; MALDI-TOF-MS (dithranol) calcd for $\mathrm{C}_{393} \mathrm{H}_{428} \mathrm{~N}_{8} \mathrm{O}_{72} \mathrm{Si}_{8} ; 6640$ (averaged); found: $\mathrm{m} / \mathrm{z} 6664\left(\sim[\mathrm{M}+\mathrm{Na}]^{+}\right) ; 6679\left(\sim[\mathrm{M}+\mathrm{K}]^{+}\right) ; 6750\left(\sim[\mathrm{M}+\mathrm{Ag}]^{+}\right)$. 
17: ${ }^{1} \mathrm{H}$ NMR (700 MHz, $\mathrm{CDCl}_{3}$ ): $\delta$ 7.92-7.72 (br, 32H, 16 DCPhth), 7.58-7.44 (m, 12H, $12 \mathrm{C}_{6} \mathrm{H}$ ), 7.21-6.40 (m, 240H, the other aromatic protons and $8 \mathrm{NHAc}), 5.68-5.56(\mathrm{~m}, 16 \mathrm{H}, 4 \mathrm{H3}$ ', 4

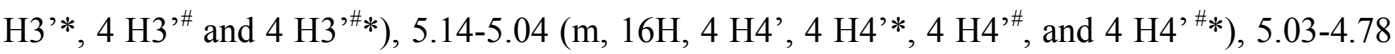
(m, 32H, $4 \mathrm{H} 1,4 \mathrm{H} 1^{\#}, 4 \mathrm{H} 4,4 \mathrm{H} 4^{\#}, 4 \mathrm{H} 1^{\prime}, 4 \mathrm{H} 1^{\prime} *, 4 \mathrm{H} 1^{\prime}{ }^{*}$ and $4 \mathrm{H} 1^{\text {, ** }}$ ), 4.70-3.93 (m, 144H, 4 $\mathrm{C} 1-\mathrm{OCH}_{2}, 4 \mathrm{Cl}^{\#}-\mathrm{OCH}_{2}, 4 \mathrm{Cl}$ '- $-\mathrm{OCH}_{2}, 4 \mathrm{Cl}$ '*- $\mathrm{OCH}_{2}, 4 \mathrm{Cl}$ ' " $-\mathrm{OCH}_{2}, 4 \mathrm{Cl}$, **-OCH, 4 C3- $\mathrm{OCH}_{2}$,

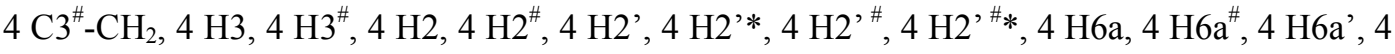
H6a'*, 4 H6a' \#, 4 H6a' **, 4 H6b, 4 H6b ${ }^{\#}, 4$ H6b', 4 H6b'*, 4 H6b' ${ }^{\text {\# }}$ and 4 H6b' **), 3.60-3.40 (m, 24H, $4 \mathrm{H} 5,4 \mathrm{H} 5^{\#}, 4 \mathrm{H} 5{ }^{\prime}, 4 \mathrm{H} 5^{*}{ }^{*}, 4 \mathrm{H} 5^{\prime}{ }^{*}$ and $\left.4 \mathrm{H} 5^{\prime}{ }^{*} *\right), 2.055,2.043,2.022,2.017,1.99$, 1.98, 1.83, 1.73, 1.721 .49 and $1.46\left(11 \mathrm{~s}, 216 \mathrm{H}, \mathrm{C}(=\mathrm{O}) \mathrm{CH}_{3}\right) ;{ }^{13} \mathrm{C} \mathrm{NMR}\left(175 \mathrm{MHz}, \mathrm{CDCl}_{3}\right): \delta$ 170.6, 170.5, 170.4, 170.1, 169.4 and $169.3\left(\underline{\mathrm{C}}(=\mathrm{O}) \mathrm{CH}_{3}\right), 165.3,139.1,130.4$ and 125.5 (DCPhth), 144.3, 141.5, 141.0, 140.9, 140.8, 140.7, 139.7, 139.5, 138.6, 133.1, 132.7, 133.1, $132.7,131.5,131.3,131.0,130.6,130.1,129.8,129.6,128.5,127.7,127.6,127.4,126.8,126.6$, 126.4, 126.3, 126.2 and 126.0 (the other aromatic carbons), 98.4 ( $\mathrm{C} 1$ and $\left.\mathrm{C} 1{ }^{\#}\right), 95.57,95.52$, 95.43 and $95.38\left(\mathrm{Cl}^{\prime}, \mathrm{C} 1^{*}, \mathrm{C} 1^{\prime *}\right.$ and $\left.\mathrm{C} 1^{*}{ }^{* *}\right), 78.4$ and $78.2\left(\mathrm{C} 3\right.$ and $\left.\mathrm{C} 3^{*}\right), 72.5\left(\mathrm{C}^{*}-\mathrm{OCH}_{2}\right.$ and $\left.\mathrm{C}^{\#}-\mathrm{OCH}_{2}\right), 71.77$ and $71.73\left(\mathrm{C} 5, \mathrm{C}^{*}, \mathrm{C} 5^{\prime}, \mathrm{C} 5^{*}, \mathrm{C} 5^{\prime \prime}\right.$ and $\left.\mathrm{C} 5^{*}{ }^{* *}\right), 70.9\left(\mathrm{C} 4\right.$ and $\left.\mathrm{C} 4^{*}\right), 70.63$ and $70.56\left(\mathrm{C}^{\prime}, \mathrm{C} 3^{\prime}{ }^{*}, \mathrm{C} 3{ }^{\prime}{ }^{*}\right.$ and $\left.\mathrm{C} 3{ }^{\prime}{ }^{*} *\right), 70.03$ and $69.98\left(\mathrm{C} 1-\mathrm{OCH}_{2}, \mathrm{Cl}^{*}-\mathrm{OCH}_{2}, \mathrm{Cl}^{\prime}-\mathrm{OCH}_{2}\right.$,

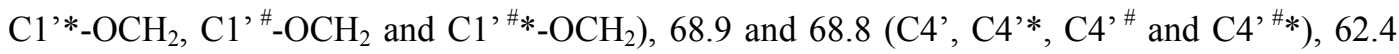

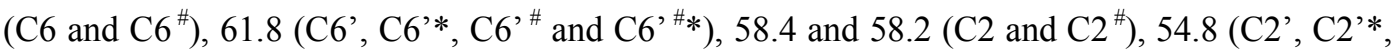
$\mathrm{C} 2^{\prime}{ }^{*}$ and $\left.\mathrm{C} 2{ }^{* * *}\right), 23.2,20.66,20.63,20.58,20.55,20.53,20.50,20.48,20.34$ and 20.28 $\left(\mathrm{C}(=\mathrm{O}) \mathrm{CH}_{3}\right)$; MALDI-TOF-MS (dithranol) calcd for $\mathrm{C}_{834} \mathrm{H}_{736} \mathrm{Cl}_{32} \mathrm{~N}_{24} \mathrm{O}_{224} ; 15813$ (averaged); found: $\mathrm{m} / \mathrm{z} 15847$.

18: ${ }^{13} \mathrm{C}$ NMR (125 MHz, $d$-DMSO): $\delta 141.6,140.7,140.2,139.2,139.0,138.82,138.78,138.4$, 136.2 , 134.9, 134.5, 130.9, 130.7, 130.0, 129.8, 129.5, 129.0, 127.6, 127.4, 126.4, 126.2, 126.0 and 125.7 (aromatic carbons), $102.2\left(\mathrm{C} 1^{\prime}, \mathrm{C} 1^{*}, \mathrm{C} 1^{*}{ }^{*}\right.$ and $\left.\mathrm{C} 1^{*}{ }^{* *}\right), 102.0\left(\mathrm{C} 1\right.$ and $\left.\mathrm{C} 1^{*}\right), 85.1(\mathrm{C} 3$

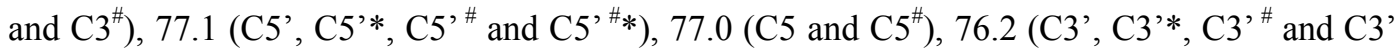
$\left.{ }^{* *}\right), 71.6\left(\mathrm{C}^{2}-\mathrm{OCH}_{2}\right.$ and $\left.\mathrm{C}^{*}-\mathrm{OCH}_{2}\right), 70.2\left(\mathrm{C}^{\prime}, \mathrm{C} 4{ }^{*}, \mathrm{C} 4{ }^{*}\right.$ and $\left.\mathrm{C} 4{ }^{*} * *\right), 69.9\left(\mathrm{C} 4\right.$ and $\left.\mathrm{C}^{*}\right), 69.1$ $\left(\mathrm{Cl}{ }^{\prime}-\mathrm{OCH}_{2}, \mathrm{Cl}{ }^{\prime}{ }_{-}-\mathrm{OCH}_{2}, \mathrm{Cl} 1^{\text {, }}-\mathrm{OCH}_{2}\right.$ and $\left.\mathrm{C1}{ }^{\text {, }}{ }^{*}-\mathrm{OCH}_{2}\right), 69.0\left(\mathrm{C} 1-\mathrm{OCH}_{2}\right.$ and $\left.\mathrm{Cl}^{*}{ }^{*}-\mathrm{OCH}_{2}\right), 61.1$

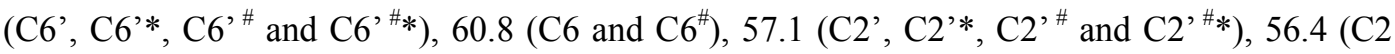
and $\mathrm{C}^{\#}$ ).

19: ${ }^{13} \mathrm{C}$ NMR (125 MHz, $d$-DMSO): $\delta 168.9\left(\mathrm{NHC}(=\mathrm{O}) \mathrm{CH}_{3}\right), 141.6,140.4,140.0,139.5,138.9$, $138.8,138.6,138.3,138.0,136.5,134.8,134.4,130.8,130.7,129.5,127.5,126.3,125.9$ and 125.6 (aromatic carbons), $102.2\left(\mathrm{Cl}^{\prime}, \mathrm{C} 1^{*}, \mathrm{C} 1^{*}{ }^{*}\right.$ and $\left.\mathrm{C} 1^{*}{ }^{*}\right), 100.5\left(\mathrm{C} 1\right.$ and $\left.\mathrm{C} 1^{*}\right), 82.7(\mathrm{C} 3$ and

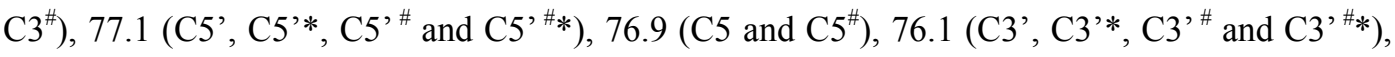
$71.5\left(\mathrm{C} 3-\mathrm{OCH}_{2}\right.$ and $\left.\mathrm{C}^{\#}-\mathrm{CH}_{2}\right), 70.1\left(\mathrm{C} 4\right.$ ', $\mathrm{C}^{\prime}{ }^{*}, \mathrm{C} 4{ }^{\prime}{ }^{*}, \mathrm{C} 4{ }^{\prime}{ }^{*} *, \mathrm{C} 4$ and $\left.\mathrm{C} 4{ }^{*}\right), 68.9\left(\mathrm{C}^{\prime}{ }^{\prime}-\mathrm{OCH}_{2}\right.$, 


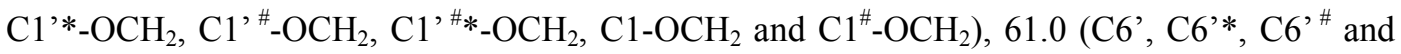

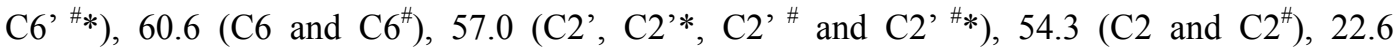
$\left(\mathrm{NHC}(=\mathrm{O}) \mathrm{CH}_{3}\right)$.

21: ${ }^{1} \mathrm{H}$ NMR (500 MHz, $\left.\mathrm{CDCl}_{3}\right): \delta$ 7.86-7.62 (m, 64H, 16 Phth), 7.50, 7.46 and $7.41(3 \mathrm{~s}, 12 \mathrm{H}$, $\left.12 \mathrm{C}_{6} \mathrm{H}\right), 7.22-6.40\left(\mathrm{~m}, 232 \mathrm{H}\right.$, the other aromatic protons), 5.79-5.70 (m, 16H, $4 \mathrm{H} 3$ ', $4 \mathrm{H} 3{ }^{\prime} *, 4$

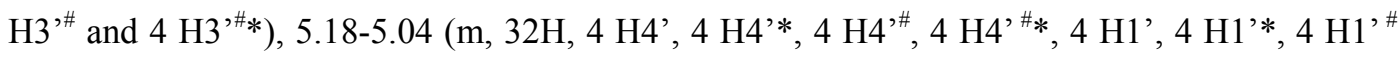

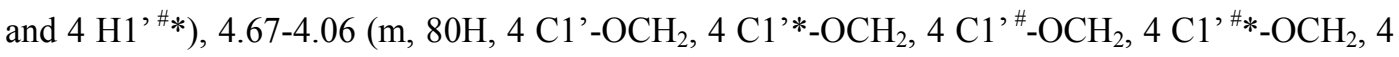

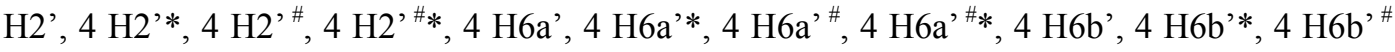

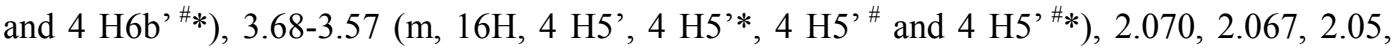
2.04, 2.034, 2.032, 2.025, 2.021, 1.85, 1.84 and $1.83\left(11 \mathrm{~s}, 144 \mathrm{H}, \mathrm{OC}(=\mathrm{O}) \underline{\mathrm{H}}_{3}\right) ; \mathrm{J}_{1}, 2,=8.2 \mathrm{~Hz}$; ${ }^{13} \mathrm{C}$ NMR $\left(125 \mathrm{MHz}, \mathrm{CDCl}_{3}\right): \delta 170.60,170.57,170.10,170.05,169.5\left(\mathrm{OC}(=\mathrm{O}) \mathrm{CH}_{3}\right), 167.4$, 134.2, 131.4 and 123.5 (Phth), 144.3, 141.8, 141.5, 141.1, 140.8, 140.7, 140.5, 139.9, 139.8, 139.7, 139.5, 139.4, 139.3, 139.1, 139.0, 138.52, 138.47, 138.1, 137.8, 133.4, 133.1, 131.8, 131.7, 131.6, 131.2, 130.4, 130.1, 129.9, 128.5, 128.3, 127.7, 127.5, 127.1, 126.7, 126.5, 126.4,

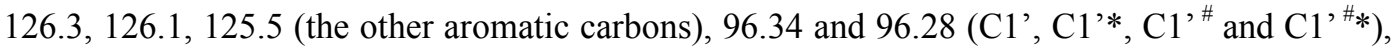

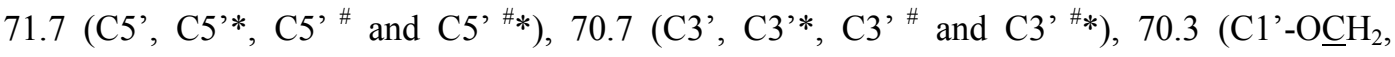

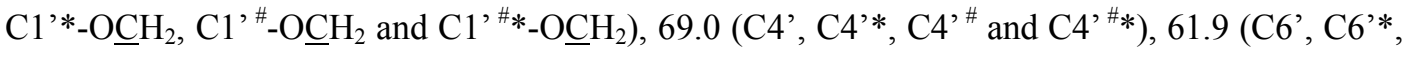
$\mathrm{C} 6^{\prime}{ }^{*}$ and $\left.\mathrm{C}^{\prime}{ }^{* *}\right), 54.50$ and $54.48\left(\mathrm{C}^{\prime}, \mathrm{C} 2^{*}, \mathrm{C} 2^{\prime}{ }^{*}\right.$ and $\left.\mathrm{C} 2^{\prime}{ }^{* *}\right), 20.7,20.6$ and 20.4 $\left(\mathrm{OC}(=\mathrm{O}) \underline{\mathrm{CH}}_{3}\right)$; MALDI-TOF-MS (dithranol) calcd for $\mathrm{C}_{721} \mathrm{H}_{596} \mathrm{~N}_{16} \mathrm{O}_{160} ; 12044$ (averaged); found: $\mathrm{m} / \mathrm{z} 12067$.

22: ${ }^{13} \mathrm{C}$ NMR (125 MHz, $d$-DMSO): $\delta$ 141.5, 141.3, 140.4, 139.9, 139.4, 139.1, 138.8, 135.1, 134.8, 130.9, 129.6, 127.8, 126.9, 126.1, 125.8 (aromatic carbons), 102.4 and 102.3 (C1', C1'*,

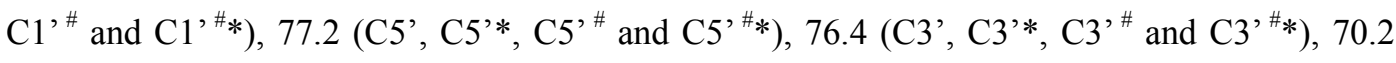

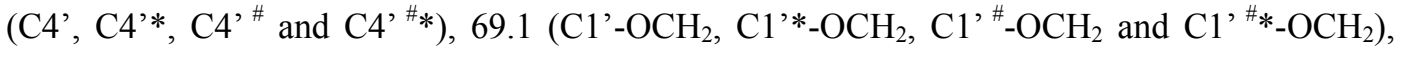

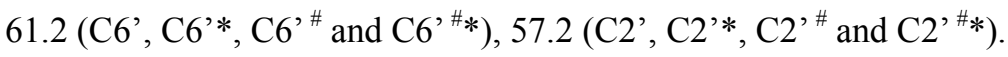

Note: 1) The peripheral sugar moieties are distinguished from the internal sugar moieties by the use of ' symbols. 2) Asymmetry generated at the first and second generation aromatic branching points places the attached sugar moieties in different environments; these are differentiated using the * and ${ }^{\#}$ symbols. 